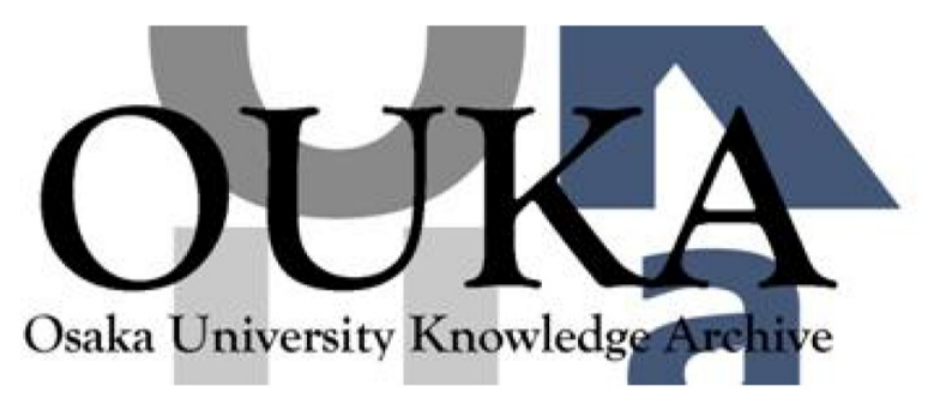

\begin{tabular}{|c|l|}
\hline Title & $\begin{array}{l}\text { SPACA1-deficient male mice are infertile with } \\
\text { abnormally shaped sperm heads reminiscent of } \\
\text { globozoospermia }\end{array}$ \\
\hline Author(s) & $\begin{array}{l}\text { Fujihara, Yoshitaka; Satouh, Yuhkoh; Inoue, } \\
\text { Naokazu et al. }\end{array}$ \\
\hline Citation & Development. 139(19) p. 3583-p.3589 \\
\hline Issue Date & $2012-10$ \\
\hline oaire:version & VoR \\
\hline URL & https://hdl. handle. net/11094/78609 \\
\hline rights & $\begin{array}{l}\text { ○ 2012. Published by The Company of Biologists } \\
\text { Ltd. }\end{array}$ \\
\hline Note & \\
\hline
\end{tabular}

Osaka University Knowledge Archive : OUKA

https://ir. Library. osaka-u. ac. jp/

Osaka University 


\title{
SPACA1-deficient male mice are infertile with abnormally shaped sperm heads reminiscent of globozoospermia
}

\author{
Yoshitaka Fujihara ${ }^{1}$, Yuhkoh Satouh ${ }^{1,2}$, Naokazu Inoue ${ }^{1}$, Ayako Isotani ${ }^{1}$, Masahito Ikawa ${ }^{1}$ and \\ Masaru Okabe ${ }^{1, *}$
}

\begin{abstract}
SUMMARY
SPACA1 is a membrane protein that localizes in the equatorial segment of spermatozoa in mammals and is reported to function in sperm-egg fusion. We produced a Spaca1 gene-disrupted mouse line and found that the male mice were infertile. The cause of this sterility was abnormal shaping of the sperm head reminiscent of globozoospermia in humans. Disruption of Spaca 1 led to the disappearance of the nuclear plate, a dense lining of the nuclear envelope facing the inner acrosomal membrane. This coincided with the failure of acrosomal expansion during spermiogenesis and resulted in the degeneration and disappearance of the acrosome in mature spermatozoa. Thus, these findings clarify part of the cascade leading to globozoospermia.
\end{abstract}

\section{KEY WORDS: Spermiogenesis, Mouse model, Acrosome}

\section{INTRODUCTION}

The acrosome is a membrane-bound cap-like structure that covers the anterior portion of the mammalian sperm nucleus. Although the size and shape of the acrosome vary considerably from species to species, its basic structure is the same in all eutherian mammals (Yanagimachi, 1994). In the testis, acrosome formation is initiated by the production of numerous proacrosomal granules in transGolgi stacks, followed by fusion into a single large acrosomal granule that associates with the nuclear envelope (Abou-Haila and Tulsiani, 2000). Subsequently, the acrosome increases in size and flattens (thinning) over the surface of the nucleus and assumes its mature species-specific conformation.

Globozoospermia is a human infertility syndrome caused by defects manifested during spermatogenesis (Dam et al., 2007a). The characteristic feature of globozoospermia is the malformation or loss of the acrosome accompanied by an abnormal nuclear shape as well as an abnormal arrangement of the sperm mitochondria (Battaglia et al., 1997). Given the tendency for a familial appearance of globozoospermia, the disease has become a target for quantitative trait locus analysis in humans (Taskiran et al., 2006).

In the mouse, various genes were found to be associated with globozoospermia by gene disruption experiments, including Csnk2a2 (Xu et al., 1999), Hrb (Agfgl - Mouse Genome Informatics) (Kang-Decker et al., 2001), Gopc (Yao et al., 2002), Gba2 (Yildiz et al., 2006), Zpbp1 (Zpbp - Mouse Genome Informatics) (Lin et al., 2007) and Pickl (Xiao et al., 2009). Interestingly, except for Zpbp1, these disrupted genes are expressed in all body tissues, but the globozoospermia phenotype was found to arise exclusively in spermatogenesis. Although all six genes seem to function at different stages of Golgi transport and acrosomal biogenesis, these proteins must be closely related

\footnotetext{
${ }^{1}$ Research Institute for Microbial Diseases and ${ }^{2}$ World Premier International Immunology Frontier Research Center, Osaka University, 3-1 Yamadaoka, Suita,
} Osaka 565-0871, Japan.

*Author for correspondence (okabe@biken.osaka-u.ac.jp)

Accepted 12 July 2012 because the phenotypes of their defects are very similar. Elucidation of the relationships among these proteins is essential to understand the molecular mechanism that leads to globozoospermia (Matzuk and Lamb, 2008). We found, serendipitously, sperm acrosome associated 1 (Spacal) to be a new member of the globozoospermia-related genes. By examining Gopc- and Zpbp1-disrupted mouse lines, we demonstrated that SPACA1 is missing in both. Evaluating the phenotypes of these mice will help elucidate the mechanism underlying globozoospermia.

\section{MATERIALS AND METHODS}

Animals

The Gopc knockout mouse strain [RBRC01253 (Yao et al., 2002)] was provided by the RIKEN BioResource Center through the National BioResource Project of MEXT, Japan. The Zpbpl knockout mouse strain was provided by Dr Martin M. Matzuk [Baylor College of Medicine, Houston, TX, USA (Lin et al., 2007)]. Wild-type mice and rats were purchased from Japan SLC (Shizuoka, Japan). All animal experiments were approved by the Animal Care and Use Committee of the Research Institute for Microbial Diseases, Osaka University, Japan.

\section{Construction of the Spaca1 gene disruption vector}

A targeting vector was constructed using pNT1.1 [http://www.ncbi.nlm.nih.gov/nuccore/JN935771 (Fujihara et al., 2010)]. A $1.5 \mathrm{~kb}$ NotI-SalI fragment as a short arm and a $1.2 \mathrm{~kb}$ EcoRI-AflII fragment including exons 3 and 4 as a middle arm and a $4.3 \mathrm{~kb}$ KpnI-AvrII fragment as a long arm were obtained by PCR amplification using genomic DNA derived from $\mathrm{C} 57 \mathrm{BL} / 6 \mathrm{~N}$ mice as a template. The PCR primers used were $\left(5^{\prime}-3^{\prime}\right)$ : TGCGGCCGCTGCCTGGAATCGGCATGAT and CGAGTCGACGTAACTGCCATGGATCTTCCC for the short arm; CGAATTCGTGTTGGTATCAGAGAAGTAA and GCTTAAGTTGGTCTGGCTTTAGAAGC for the middle arm; CGGTACCGTGAGTATTCTGTGTATAAATAATCC and GCCTAGGCCTATCACAAAGGAACTCAC for the long arm. These three fragments were inserted into a pNT1.1 vector and the targeting construct was linearized with NotI. Embryonic stem (ES) cells were electroporated and colonies were screened as described below.

\section{Generation of Spaca1-disrupted mice}

The mouse Spacal gene consists of seven exons and maps to chromosome 4. The targeting vector was designed to remove the third and fourth exons of Spacal (supplementary material Fig. S2A) and was electroporated into EGR-G01 [129S2 × $(\mathrm{cag} /$ acr-EGFP $) \mathrm{C} 57 \mathrm{BL} / 6 \mathrm{~N} \mathrm{F1]}$ ES cells after 
linearization. Potentially targeted ES cell clones were separated by positive/negative selection with G418 and ganciclovir. Correct targeting of the Spacal allele in ES cell clones and germline transmission were determined by PCR analysis. Screening primers were $\left(5^{\prime}-3^{\prime}\right)$ : CACAGGGAAGCTGCTCGTG and GCTTGCCGAATATCATGGTGGAAAATGGCC for the short arm; CAGCCTCTGAGCCCAGAAAGCG and AGGCTGCAAATAACCCAGATGC for the middle and long arms.

Spacal-floxed mice obtained from germline transmission mouse chimeras had normal fertility. For the removal of loxP-flanked exons, mating between $\mathrm{F} 1$ mice and $C A G-C r e$ transgenic mice $[\mathrm{B} 6 . \mathrm{Cg}-\mathrm{Tg}(C A G-$ $\mathrm{Cre}) \mathrm{CZ}-\mathrm{MO} 2 \mathrm{Osb}]$ yielded offspring that carried a heterozygous deficiency of the Spacal gene (supplementary material Fig. S2A). Mating between heterozygous mutant mice yielded the expected Mendelian ratios: Spacal $^{+++}, 22.7 \%$; Spacal $1^{+/}, 50.0 \%$; Spacal $1^{-/}, 27.3 \%$ of offspring $(n=66)$. Genotyping primers were $\left(5^{\prime}-3^{\prime}\right)$ : GGGAGGGAAGATCCATGGCA and GCGCTAACACTCATTGATCTGGC. Both a $1.6 \mathrm{~kb}$ band for the wildtype allele and a $0.5 \mathrm{~kb}$ band for the mutant allele were amplified by PCR (supplementary material Fig. S2B). Western blot analysis showed that SPACA1 protein was undetectable in $\mathrm{Spacal}^{-/-}$testis (supplementary material Fig. S2C). Spaca1 ${ }^{-/}$mice were healthy and showed no overt developmental abnormalities. Mice used in this study were of a B6;129 mixed background.

The Spacal-disrupted mouse line has been submitted to the RIKEN BioResource Center (http://www.brc.riken.jp/inf/en/index.shtml) and is available to the scientific community.

\section{Assessment of the fertility of SPACA1-deficient mice}

Sexually mature male mice of genotypes Spacal ${ }^{+/+}$, Spacal $^{+/-}$and Spaca1 $^{-/-}$were caged with $(\mathrm{C} 57 \mathrm{BL} / 6 \mathrm{~N} \times \mathrm{DBA} / 2 \mathrm{~N}) \mathrm{F} 1$ (also known as B6D2F1) female mice (at least 2 months old; CLEA Japan, Tokyo, Japan) for 2 months and the number of pups in each cage was counted within a week of birth.

\section{Antibodies}

Rabbit anti-mouse SPACA1 polyclonal antiserum was produced by immunization with mouse SPACA1 polypeptide (QSPTDIPVHEDDALSEWNE). A polyclonal antibody against human SPACA1 (\#BP5112, C-terminal domain: CGEDDALSEWNE) was purchased from Acris Antibodies (Herford, Germany). A rabbit anti-GOPC polyclonal antibody was a gift from Dr Tetsuo Noda [The Cancer Institute of the Japanese Foundation for Cancer Research, Tokyo, Japan (Yao et al., 2001)]. A rabbit anti-ZPBP1 antibody (G176) was a gift from Dr Martin M. Matzuk (Lin et al., 2007). The mouse monoclonal antibody MN9 was a gift from Dr Kiyotaka Toshimori [Chiba University, Chiba, Japan (Toshimori et al., 1992)]. A goat anti-basigin (CD147) polyclonal antibody was purchased from Santa Cruz Biotechnology (Santa Cruz, CA, USA). A polyclonal antibody against mouse calnexin and the monoclonal antibody KS64-10 (for SLC2A3) were established previously in our laboratory according to standard methods (Ikawa et al., 2001; Yamaguchi et al., 2006).

\section{Immunohistochemistry}

Immunohistochemistry was performed as described previously for the testicular germ cells shown in Fig. 1D and supplementary material Fig. S1 (Kotaja et al., 2004) and for the epididymal spermatozoa shown in Fig. 1E (Yamaguchi et al., 2008) using a fluorescence microscope (Olympus BX50).

\section{Electron microscopy}

Scanning electron microscopy (SEM) (Fujihara et al., 2010) and transmission electron microscopy (TEM) (Inoue et al., 2011) were performed as described previously.

\section{Immunoblot analysis and PNGase $\mathrm{F}$ treatment}

Immunoblot analyses (Inoue et al., 2008), extraction of human sperm proteins (Mandal et al., 1999) and phase separation of Triton X-114 extracts of sperm proteins (Yamaguchi et al., 2006) were performed as described. All samples were separated by SDS-PAGE under reducing conditions. Peptide-N-glycosidase F (PNGase F) (Roche Applied Science, Germany) treatment was performed as described (Inoue et al., 2008). Testis lysates were treated with $250 \mathrm{mU}$ PNGase $\mathrm{F}$ for 16 hours at $37^{\circ} \mathrm{C}$ according to the manufacturer's instructions. The treated lysates were subjected to SDS-PAGE followed by western blotting.

\section{RT-PCR}

RT-PCR analysis using testicular cDNA was performed as described (Fujihara et al., 2010) using the following primers $\left(5^{\prime}-3^{\prime}\right)$ : GGCGGCAGTGAAATCCTTCT and TGCTCTAATGGTCTCTGGTTTCTC for Spacal; CCACTCAGCTTCAGCTTCATGCC and AGCCTGGAGAACAGCTATGTGCC for Gopc; TTGGACATTTGGCTCGACTGCC and CCTTTTGGCCCATGCCATTGG for Zpbp 1; ACATTCACGGAAGCGCTGGG and ACGGTGTTCTCAGCACAAGGC for Csnk2a2; AGTTTCCAGCAGCCTGCCTTC and GAGCTTCCTGTTGGAAGTTGTCCT for $H r b$; CTTTGCAGCAAAGGAGCAGCTC and CGGCTGCCTGACGAAGATGG for Spatal6; GGCTCGAGCCCGCTTCTC and GCCTTGGTTAGGGCCATAGGC for Pick1; AAGTGTGACGTTGACATCCG and GATCCACATCTGCTGGAAGG for $\beta$-actin $(A c t b)$

The amplification conditions were 1 minute at $96^{\circ} \mathrm{C}$, followed by 40 cycles (for Fig. 1A) or 27 cycles (for Fig. $4 \mathrm{~A}$ ) of $96^{\circ} \mathrm{C}$ for 30 seconds, $65^{\circ} \mathrm{C}$ for 30 seconds, and $72^{\circ} \mathrm{C}$ for 30 seconds, with a final 1 -minute extension at $72^{\circ} \mathrm{C}$. The expression of Scp 3 (Sycp3 - Mouse Genome Informatics) and Prm1, which were used as markers for the meiotic spermatocyte and the spermatid stages, respectively, had been established previously (Fujihara et al., 2010).

\section{RESULTS}

\section{Expression and localization of mouse SPACA1}

The expression of Spacal in various organs was examined by RTPCR. Spacal was exclusively expressed in testis and the SPACA1 protein was found in spermatozoa as previously reported in human (Hao et al., 2002), boar, ram and bull (Jones et al., 2008) (Fig. 1A,B). Presence of SPACA1 was demonstrated by western blotting after proteins had been extracted from spermatozoa using Triton X114 treatment followed by phase separation and SDS-PAGE. SPACA1 was detected in the detergent-enriched phase, indicating that SPACA1 is a transmembrane protein (Fig. 1C).

Immunostaining over a range of developmental stages (supplementary material Fig. S1) revealed that SPACA1 first appears at the step 2 stage of round spermatids and was localized exclusively on a peripheral part of the acrosomal membrane, forming a ring shape in step 7 spermatids (Fig. 1D). After the sperm head and acrosome had elongated, SPACA1 became localized on the equatorial segment of the acrosome. SPACA1, being a transmembrane protein, was not released from spermatozoa even after exocytosis of the acrosomal contents in the acrosome reaction, which is essential for spermatozoa to fertilize eggs (Fig. 1E).

\section{Production of Spaca1 null mice}

Spacal was disrupted by homologous recombination (supplementary material Fig. S2A). RT-PCR analysis confirmed the successful elimination of the Spacal gene and western blot analysis demonstrated the absence of SPACA1 in Spacal ${ }^{-/}$testes (supplementary material Fig. S2B,C). To examine the fertilizing ability of Spacal-disrupted mouse line, three to five adult males of each genotype were bred with wild-type females for 2 months. Whereas Spacal ${ }^{+/-}$mice showed similar fertility to Spacal $^{+/+}$mice, the Spacal $^{-/}$males were completely sterile (Fig. 1F). The mean litter size in Spaca1 ${ }^{+/+}$males was 9.6 $\pm 1.6(n=15)$ and in Spacal ${ }^{+/-}$ males it was $8.7 \pm 2.5(n=23)$. The testicular weights of Spacal ${ }^{-/}$ $(109.5 \pm 12.9 \mathrm{mg} ; n=12)$ and Spacal $^{+/+}(113.5 \pm 11.2 \mathrm{mg} ; n=12)$ mice were similar (supplementary material Fig. S2D). Spacal ${ }^{-1}$ females were fertile (Fig. 1F; mean litter size $8.7 \pm 2.6 ; n=23$ ), reflecting the fact that Spacal is not expressed in female reproductive organs. 
A

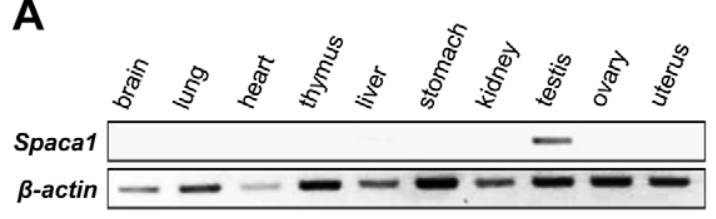

B

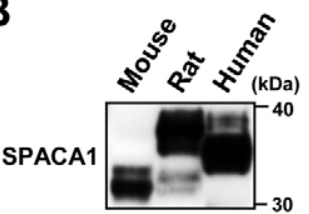

C

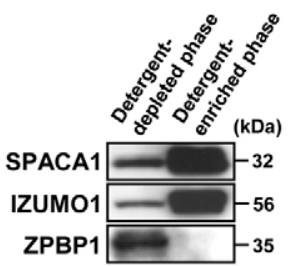

E

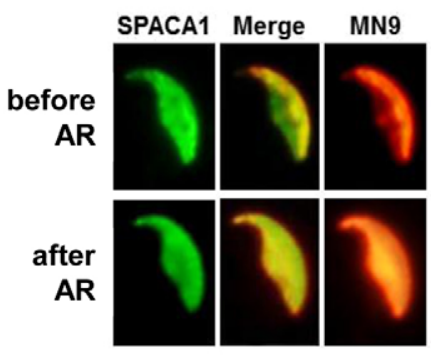

D

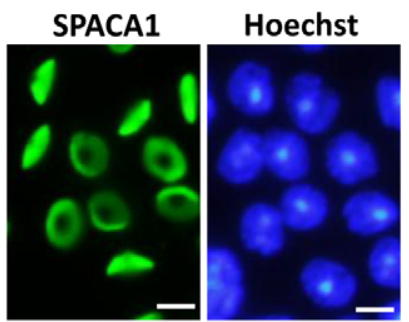

F

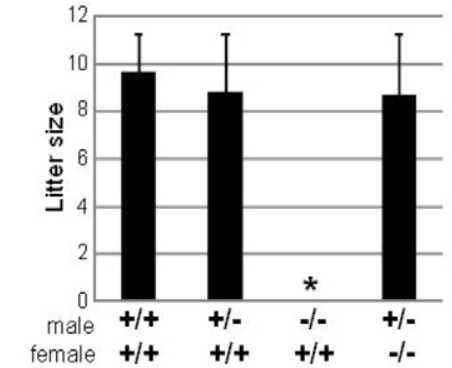

Fig. 1. Characterization of SPACA1 and the phenotype of the Spaca1-disrupted mouse line. (A) Testis-specific expression of Spaca1 by multi-tissue RT-PCR analysis. The expression of mouse Spaca1 was examined by RT-PCR using RNA isolated from various organs. Spaca1 was expressed only in the testis. The $\beta$-actin gene was used as an expression level control. (B) SPACA1 protein in mouse, rat and human spermatozoa. Spermatozoa were extracted with Triton X-100 and detected using an anti-SPACA1 polyclonal antibody (\#BP5112). (C) Sperm proteins were phase separated following extraction with $1 \%$ Triton X-114. SPACA 1 was found predominantly in the detergent-enriched fraction, as in the case of the membrane protein IZUMO1, whereas the soluble protein ZPBP1 was found only in the detergent-depleted fraction. (D) SPACA1 in step 7 round spermatids immunostained by anti-SPACA1 polyclonal antibody and anti-rabbit antibody (green). Nuclei were stained with Hoechst 33342 (blue) Scale bars: $5 \mu \mathrm{m}$. (E) Immunofluorescence staining of SPACA1 before and after the acrosome reaction (AR). SPACA1 was found in the equatorial segment in spermatozoa before and after the AR. The monoclonal antibody MN9 was used to demonstrate acrosomal integrity. Crescentic and equatorial segment staining patterns indicate spermatozoa before and after the AR (Yoshida et al., 2010). (F) Fertility of SPACA1-deficient mice. Spermatozoa from Spaca $1^{-1-}$ mice were completely sterile (asterisk). Error bars indicate s.d.

\section{Impaired spermatogenesis in the SPACA1- deficient mouse}

The swimming ability of Spaca1 ${ }^{-/}$spermatozoa was significantly impaired (supplementary material Movie 1). Moreover, when the Spacal $^{-/}$spermatozoa were examined under a fluorescence microscope, all of the testicular spermatozoa showed a roundheaded morphology (Fig. 2A), and spermatozoa from the cauda epididymidis showed a tail coiled around the head (Fig. 2B; supplementary material Fig. S2F). To analyze acrosomal integrity, Spacal $^{-/}$males were mated with Acr-EGFP transgenic mice (Nakanishi et al., 1999) in which acrosomes can be visualized by enhanced green fluorescence (EGFP). All of the round-headed spermatozoa from Spaca1 ${ }^{-/}$mice had an acrosome of negligible size (Fig. 2A,B). A few of the Spacal ${ }^{-/}$spermatozoa $(<5 \%)$ had small acrosomes of aberrant shape.

These abnormalities were also examined by SEM and TEM. Disruption of Spacal caused hypoplasia of intermediate filament bundles, which normally form a characteristic dense structure on the nuclear membrane facing the inner acrosomal membrane (Fig. 2C,D) (Ito et al., 2004). Thinning of the acrosome was not observed in Spacal ${ }^{-/}$spermatids. Mature spermatozoa had disorganized mitochondria trapped inside large cytoplasmic droplets, misshapen nuclei and ectopic localization of the flagella
(Fig. 2E,F). The Spacal $^{-/-}$spermatozoa were seen to be roundheaded in SEM analysis, in contrast to the hook-shaped spermatozoa observed in wild type (Fig. 2F). These abnormalities are reminiscent of the defects seen in the human condition globozoospermia (Dam et al., 2007a). Similar to Gopc null (Suzuki-Toyota et al., 2007) and Zpbp1 null (Lin et al., 2007) mice, this morphological aberrance became more evident while the spermatozoa were passing through the epididymis. Although these globozoospermic spermatozoa are abnormal in appearance and exhibit no ability to fertilize eggs by themselves, when the sperm head is introduced into an egg by intracytoplasmic sperm injection (ICSI), healthy pups can be obtained (Dam et al., 2007a; Yao et al., 2002). In our studies, we demonstrated that Spaca $1^{-/}$spermatids can produce healthy pups by performing round spermatid injection (ROSI) into eggs (supplementary material Fig. S3).

\section{Acrosomal status in SPACA1-deficient spermatozoa}

Abnormal spermatogenesis was not obvious in routine histology sections prepared from Spacal ${ }^{-/}$testes stained with Hematoxylin and Eosin (supplementary material Fig. S2E). We crossed the Spacal ${ }^{-/-}$male mice with transgenic mice expressing EGFP in their acrosome so that acrosome formation during spermatogenesis 


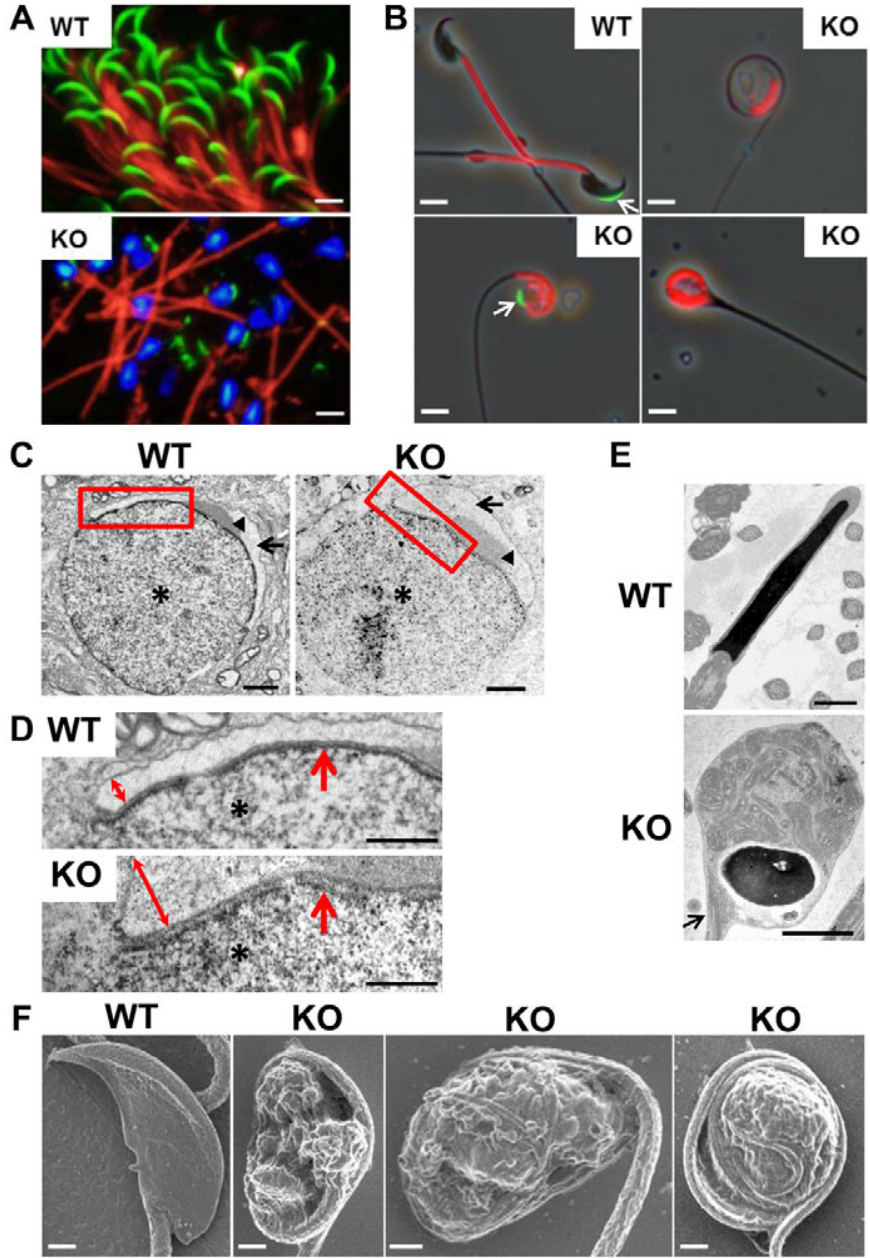

Fig. 2. Malformation of spermatozoa in SPACA1-deficient mice.

(A) Fluorescence microscopy of testicular spermatozoa in wild-type (WT) and Spaca1 knockout (KO) mice. Green and red fluorescence show transgenically expressed EGFP in the acrosome and red fluorescent protein (RFP DsRed2) in mitochondria, respectively (Hasuwa et al., 2010). Nuclei were stained with Hoechst 33342 (blue). (B) Phase-contrast microscopy of spermatozoa from the cauda epididymidis. Green fluorescence shows transgenically expressed EGFP in the acrosome (arrows). Red fluorescence indicates mitochondria (stained with MitoTracker Red CMXRos, Invitrogen). (C) A comparative TEM view of SPACA1-deficient and wild-type round spermatids. Acrosomes (arrows) failed to stretch out along with the nuclear surface when Spaca1 was disrupted. The acrosomal granule and sperm nucleus are indicated by arrowhead and asterisk, respectively. Staging of spermatids was estimated based on the degree of nuclear condensation. (D) Enlarged views of the boxed area in C. No close association of the acrosomal membrane with the nuclear envelope or with intermediate filament bundles was formed in SPACA1-deficient spermatids. The nuclear plate, a dense lining of the nuclear envelope, was also missing (arrows) and the acrosome (double arrows) failed to proceed to the thinning stage in SPACA1-deficient spermatids. The nucleus is indicated by an asterisk. (E) A representative view of wild-type and SPACA1-deficient spermatozoa from the cauda epididymidis. SPACA1-deficient spermatozoa had a globular nucleus and congregated mitochondria. The arrow indicates the beginning of the flagellum. (F) SEM of wild-type and SPACA1-deficient spermatozoa. Scale bars: $5 \mu \mathrm{m}$ in $\mathrm{A}, \mathrm{B} ; 1 \mu \mathrm{m}$ in $\mathrm{C}, \mathrm{E}, \mathrm{F} ; 0.5 \mu \mathrm{m}$ in $\mathrm{D}$.

could be examined easily by fluorescence microscopy (Nakanishi et al., 1999). We found that the acrosome was shaped normally until around step 3 of spermatogenesis (Fig. 3). The amount of

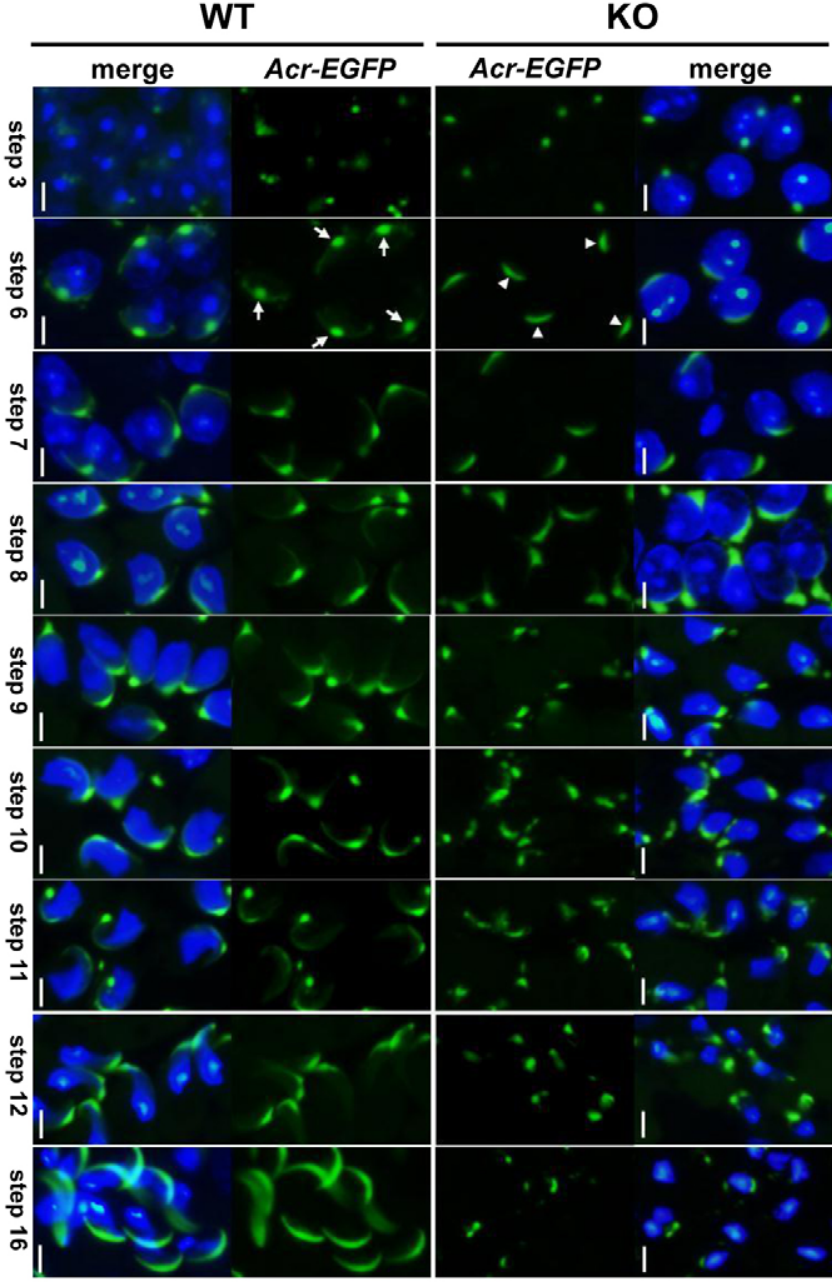

Fig. 3. Stepwise observation of acrosome formation in SPACA1deficient males. Acrosome formation was examined using spermatozoa expressing EGFP in the acrosome. Accumulation of EGFP in the acrosomal granule in step 6 spermatids (arrows) was not observed in SPACA1-deficient spermatids. EGFP was distributed uniformly in the SPACA1-deficient sperm acrosome (arrowheads). Staging of spermiogenesis after this step was estimated based on the degree of nuclear condensation. The accumulation of EGFP in the acrosome stopped around step 7 of spermatid formation in the SPACA1-deficient mouse line, demonstrating impaired spermiogenesis. After step 8, the acrosome started to degenerate and disappear in many of the spermatids. Nuclei were stained with Hoechst 33342 (blue). Scale bars: $5 \mu \mathrm{m}$.

EGFP (estimated from its brightness) transferred from the Golgi apparatus to the acrosome in Spacal ${ }^{-/-}$mice also seemed to be equivalent to that in wild-type spermatids, at least in the early steps of spermatogenesis (Fig. 3). However, the abnormality became evident in spermatids at around step 6. By this stage, accumulation of EGFP was observed in the acrosomal granule in wild-type spermatids together with a thinly extended peripheral part of the acrosome (Fig. 3, arrows). In Spacal ${ }^{-1}$ spermatids, by contrast, the accumulation of GFP in the acrosomal granule was not apparent and the acrosomal extension was insufficient to form a thin peripheral structure (Fig. 3, arrowheads). Formation of the acrosome did not progress further after this step and in most of the spermatids it degenerated and disappeared (Fig. 3). Differences in nuclear shape were also noted. The nuclei of $\mathrm{Spacal}^{-/-}$spermatids 
A
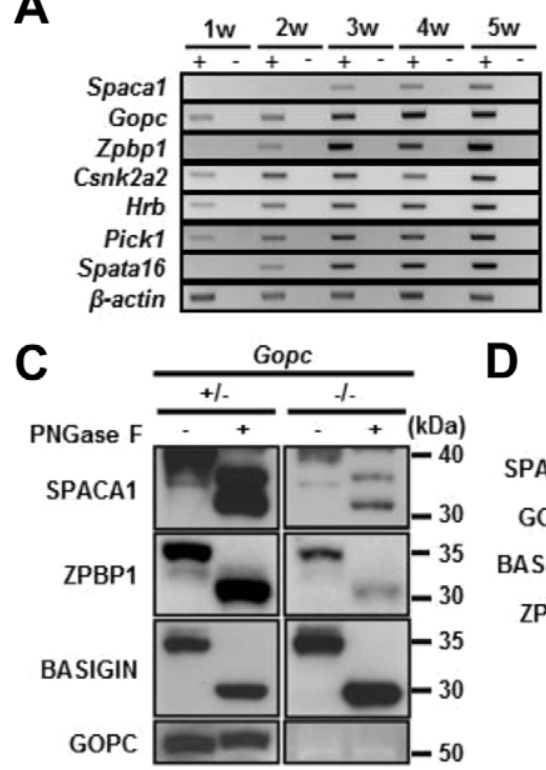

D
B
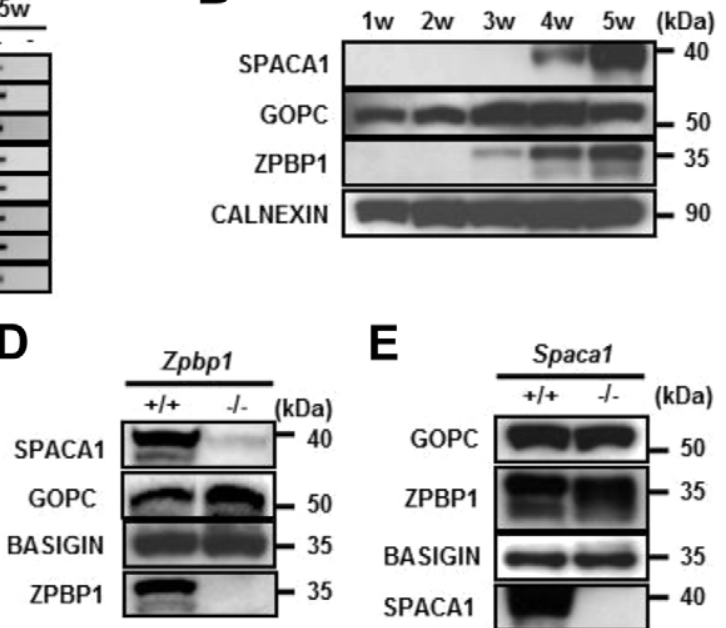

E

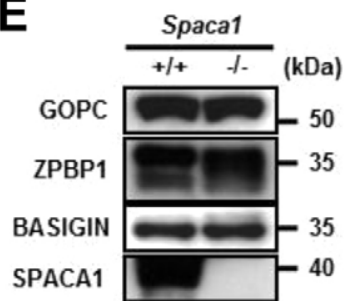

Fig. 4. Timing of expression of SPACA1 and other globozoospermia-related proteins in the testis. (A) RT-PCR analysis of Spaca1 and other globozoospermia-related genes in the testis. Expression of Spaca1 was first detected in 3-week-old mouse testes. The onset of expression of all other globozoospermia-related genes tested occurred prior to that of Spaca1. The $\beta$-actin gene was used as an expression control. (B) SPACA1 was first detectable in the 4-week-old testis of ICR mice. GOPC and ZPBP1 were first detectable at an earlier stage of spermatogenesis. Calnexin was used as a loading control. (C) PNGase F treatment lowered the molecular weights of both SPACA1 and ZPBP1 in the testis. Basigin was used as a control. Note that the disruption of Gopc caused a significant decrease in SPACA1 and ZPBP1. (D) Disruption of Zpbp1 caused the loss of SPACA1 whereas GOPC was unaffected. (E) Disruption of Spaca1 did not affect the amounts of GOPC and ZPBP1 in the testis. Immunoblotting (B-E) was performed using $20 \mu \mathrm{g}$ aliquots of Triton X-100 extracts from wild-type, GOPC-deficient, ZPBP1-deficient and SPACA1-deficient testes, using basigin as a loading control.

were smaller than those of wild-type spermatids, probably because they remained globular rather than increasing their apparent area by thinning as in wild-type spermatids (Fig. 2E,F).

\section{Expression of Spaca1 in other globozoospermia- related gene disruption mouse lines}

To clarify the functional and temporal assembly of the acrosome with respect to the components expressed at different stages of acrosomal biogenesis, the onset of Spacal expression was compared by RT-PCR (Fig. 4A) and western blotting (Fig. 4B) with that of other globozoospermia-related genes: Csnk2a2 (Xu et al., 1999), Hrb (Kang-Decker et al., 2001), Gopc (Yao et al., 2002), Zpbpl (Lin et al., 2007), Spata16 (Dam et al., 2007b) and Pick1 (Xiao et al., 2009). Spacal expression was the last to appear among all the strains examined (Fig. 4A). SPACA1 protein was detectable only after 4 weeks of age in these mice (Fig. 4B). Using PNGase F treatment, SPACA1 was shown to be glycosylated, as is the case for ZPBP1 (Lin et al., 2007) (Fig. 4C). It should be noted that the amounts of SPACA1 and ZPBP1 were decreased significantly in the Gopc-disrupted testis (Fig. 4C). By contrast, the amount of GOPC was unaffected by the disruption of either Zpbp1 or Spacal (Fig. 4D,E). Likewise, disruption of Zpbpl caused the loss of SPACA1 (Fig. 4D), but ZPBP1 expression remained unchanged in the Spacal-disrupted testis (Fig. 4E). These results indicate that SPACA1 must be functioning further downstream than any of the other key molecules associated with globozoospermia.

\section{DISCUSSION}

In eutherian mammals, the equatorial segment of the sperm head is the site where sperm-egg fusion initiates (Bedford and Cooper,
1978). To date, IZUMO1 is the only sperm factor reported to be essential for fusion, but it is localized over the entire head (Inoue et al., 2005). For this reason, we were actively seeking a fusion factor localized only in the equatorial segment. It was reported that SPACA1 (SAMP32) is localized in this region (Hao et al., 2002; Jones et al., 2008) and that an anti-SPACA1 antibody could inhibit the fusion of human spermatozoa with zona-free hamster eggs (Hao et al., 2002). Therefore, to investigate the role of SPACA1 in sperm-egg fusion, we produced a Spacal-disrupted mouse line. The male mice were infertile, as we expected, but the major cause of this sterility was found to be an abnormal shaping of spermatozoa reminiscent of human globozoospermia.

Because the acrosome is a large Golgi-derived secretory vesicle containing a variety of enzymes, it was unsurprising to find that many of the factors (CSNK2A2, HRB, GOPC and PICK1) that are associated with globozoospermia function in protein transport to the Golgi apparatus and/or acrosome. However, SPACA1 is an integral acrosomal membrane protein, indicating that the major cause of globozoospermia is not aberrant protein transport in the Golgi or into the acrosome. Rather, the globozoospermia in Gopcand Zpbp1-disrupted mouse lines is due to the loss of SPACA1 from their acrosomes (Fig. 4C,D).

Disruption of Spacal led to poor formation of intermediate filament bundles, which normally form a characteristic dense structure on the nuclear membrane facing the inner acrosomal membrane (Ito et al., 2004) (Fig. 2C,D). We presume that this leads both to the failure of thinning of the acrosome and to the impaired formation of the acroplaxome, an F-actin/keratin-containing plate that separates the inner acrosomal and nuclear membranes (supplementary material Fig. S4). At present, no apparent 


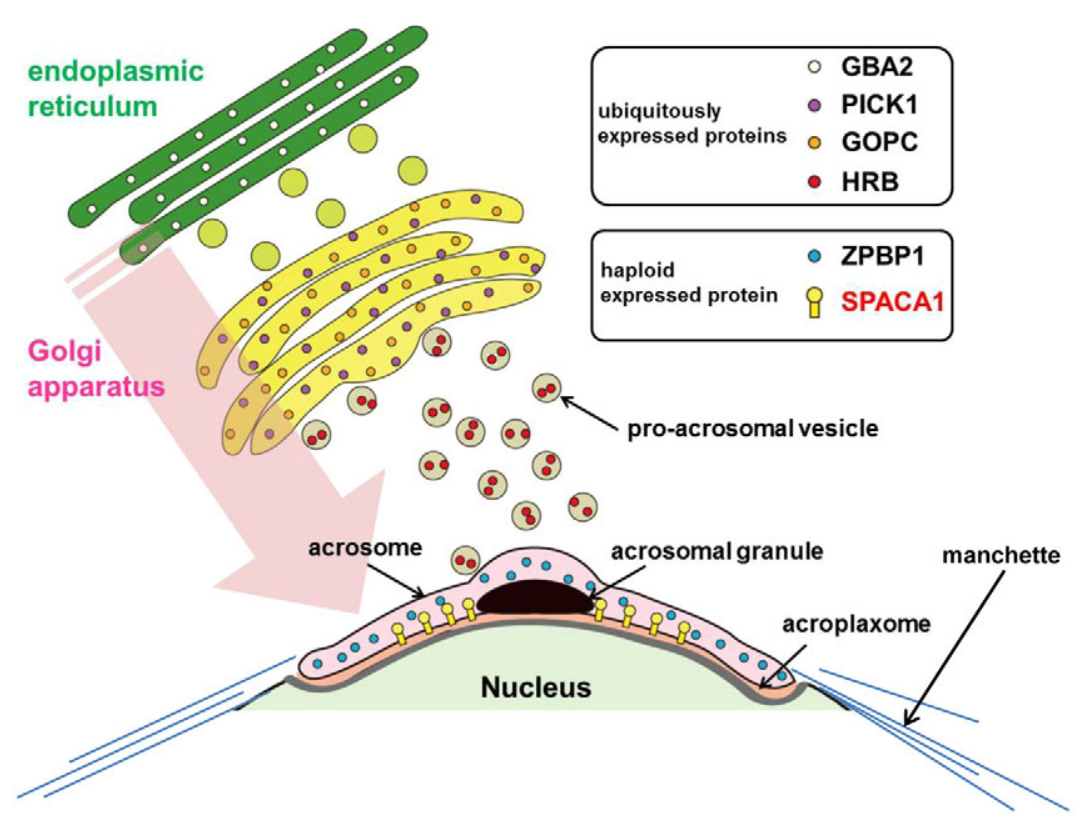

Fig. 5. Proteins associated with globozoospermia in the mouse. GBA2 is a ubiquitously expressed resident enzyme of the endoplasmic reticulum (Yildiz et al., 2006), PICK1 is a membrane protein (Xiao et al., 2009) that is considered to function in protein trafficking, GOPC is a Golgi-associated protein (Yao et al., 2002) and HRB is reported to be crucial for the docking and/or fusion of Golgi-derived proacrosomal vesicles to the acrosome (Kang-Decker et al., 2001). All of these proteins are expressed ubiquitously in somatic cells (except for PICK1 in nerve cells), but gene disruption experiments revealed defects in spermatogenesis. SPACA1 is a membrane protein that localizes in the inner acrosomal membrane. SPACA1 deficiency causes the loss of the nuclear plate, a dense structure overlying the nuclear envelope (see Fig. 2D). Loss of the nuclear plate was characteristic of Spaca $1^{-1-}$ germ cells, which implies the importance of this structure in acrosome formation and in the development of globozoospermia.

functional domain has been found in the SPACA1 protein. However, according to Jones et al. (Jones et al., 2008), SPACA1 possesses a potential Y268 tyrosine phosphorylation site that is conserved in all mammalian sequences. This might indicate that the function of SPACA1 is regulated by phosphorylation.

Kierszenbaum and Tres (Kierszenbaum and Tres, 2004) hypothesized that, during spermatogenesis, a marginal ring that forms at the edge of the acroplaxome reduces its diameter as it descends gradually along the elongating spermatid. Apparently, this process coincides with the extension of the acrosomal area attaching to the elongating spermatid nucleus. Our observations are in accordance with the above hypothesis, so impaired acrosomeacroplaxome-manchette formation might be involved in the cascades that lead to globular-headed spermatozoa (Fig. 5). This impaired complex formation did not seem to cause severe abnormalities in mitochondrial shape and sperm tail formation during spermatogenesis, but abnormal coiling of the tail around the sperm head together with misshapen mitochondria became prominent during sperm passage thorough the epididymis (Fig. 2A,B). The same phenomenon was also reported in both Gopc- and Zpbp1-disrupted spermatozoa (Lin et al., 2007; Suzuki-Toyota et al., 2007; Yao et al., 2002). The reasons for the progression of such abnormalities in the epididymis remain to be clarified.

SPACA1 is present in human spermatozoa and SPACA1 content is reported to be decreased in patients with globozoospermia as assessed by proteomic analysis (Liao et al., 2009). Further work will be required to clarify the mechanism of deficiency of SPACA1 leading to globozoospermia.

\section{Acknowledgements}

We thank Dr Tetsuo Noda at The Cancer Institute of the Japanese Foundation for Cancer Research for kindly providing the Gopc knockout mouse line and anti-GOPC polyclonal antibody; Dr Martin M. Matzuk at Baylor College of Medicine for kindly providing the Zpbp1 knockout mouse line and anti-ZPBP1 polyclonal antibody; and Akiko Kawai, Yumi Koreeda and Yoko Esaki for technical assistance in producing transgenic mouse lines.

\section{Funding}

This work was supported by grants from the Ministry of Education, Culture, Sports, Science, and Technology of Japan.
Competing interests statement

The authors declare no competing financial interests.

\section{Supplementary material}

Supplementary material available online at

http://dev.biologists.org/lookup/suppl/doi:10.1242/dev.081778/-/DC1

\section{References}

Abou-Haila, A. and Tulsiani, D. R. (2000). Mammalian sperm acrosome: formation, contents, and function. Arch. Biochem. Biophys. 379, 173-182.

Battaglia, D. E., Koehler, J. K., Klein, N. A. and Tucker, M. J. (1997). Failure of oocyte activation after intracytoplasmic sperm injection using round-headed sperm. Fertil. Steril. 68, 118-122.

Bedford, J. M. and Cooper, G. W. (1978). Membrane fusion events in fertilization of vertebrate eggs. In Membrane Surface Reviews (Membrane Fusion), Vol. 5 (ed. G. Poste and G. L. Nicolson), pp. 65-125. Amsterdam: Elsevier Science.

Dam, A. H., Feenstra, I., Westphal, J. R., Ramos, L., van Golde, R. J. and Kremer, J. A. (2007a). Globozoospermia revisited. Hum. Reprod. Update 13 63-75

Dam, A. H., Koscinski, I, Kremer, J. A., Moutou, C, Jaeger, A. S., Oudakker A. R., Tournaye, H., Charlet, N., Lagier-Tourenne, C., van Bokhoven, H. et al. (2007b). Homozygous mutation in SPATA16 is associated with male infertility in human globozoospermia. Am. J. Hum. Genet. 81, 813-820.

Fujihara, Y., Murakami, M., Inoue, N., Satouh, Y., Kaseda, K., Ikawa, M. and Okabe, M. (2010). Sperm equatorial segment protein 1, SPESP1, is required for fully fertile sperm in mouse. J. Cell Sci. 123, 1531-1536.

Hao, Z., Wolkowicz, M. J., Shetty, J., Klotz, K., Bolling, L., Sen, B., Westbrook, V. A., Coonrod, S., Flickinger, C. J. and Herr, J. C. (2002)

SAMP32, a testis-specific, isoantigenic sperm acrosomal membrane-associated protein. Biol. Reprod. 66, 735-744

Hasuwa, H., Muro, Y., Ikawa, M., Kato, N., Tsujimoto, Y. and Okabe, M. (2010). Transgenic mouse sperm that have green acrosome and red mitochondria allow visualization of sperm and their acrosome reaction in vivo. Exp. Anim. 59, 105-107.

Ikawa, M., Nakanishi, T., Yamada, S., Wada, I., Kominami, K., Tanaka, H., Nozaki, M., Nishimune, Y. and Okabe, M. (2001). Calmegin is required for fertilin alpha/beta heterodimerization and sperm fertility. Dev. Biol. 240, 254261.

Inoue, N., Ikawa, M., Isotani, A. and Okabe, M. (2005). The immunoglobulin superfamily protein Izumo is required for sperm to fuse with eggs. Nature $\mathbf{4 3 4}$ 234-238.

Inoue, N., Ikawa, M. and Okabe, M. (2008). Putative sperm fusion protein IZUMO and the role of N-glycosylation. Biochem. Biophys. Res. Commun. 377, 910-914.

Inoue, N., Satouh, Y., Ikawa, M., Okabe, M. and Yanagimachi, R. (2011) Acrosome-reacted mouse spermatozoa recovered from the perivitelline space can fertilize other eggs. Proc. Natl. Acad. Sci. USA 108, 20008-20011.

Ito, C., Suzuki-Toyota, F., Maekawa, M., Toyama, Y., Yao, R., Noda, T. and Toshimori, K. (2004). Failure to assemble the peri-nuclear structures in GOPC 
deficient spermatids as found in round-headed spermatozoa. Arch. Histol. Cytol. 67, 349-360

Jones, R., James, P. S., Oxley, D., Coadwell, J., Suzuki-Toyota, F. and Howes,

E. A. (2008). The equatorial subsegment in mammalian spermatozoa is enriched in tyrosine phosphorylated proteins. Biol. Reprod. 79, 421-431.

Kang-Decker, N., Mantchev, G. T., Juneja, S. C., McNiven, M. A. and van

Deursen, J. M. (2001). Lack of acrosome formation in Hrb-deficient mice. Science 294, 1531-1533.

Kierszenbaum, A. L. and Tres, L. L. (2004). The acrosome-acroplaxomemanchette complex and the shaping of the spermatid head. Arch. Histol. Cytol. 67, 271-284

Kotaja, N., Kimmins, S., Brancorsini, S., Hentsch, D., Vonesch, J. L., Davidson, I., Parvinen, M. and Sassone-Corsi, P. (2004). Preparation, isolation and characterization of stage-specific spermatogenic cells for cellular and molecular analysis. Nat. Methods 1, 249-254.

Liao, T. T., Xiang, Z., Zhu, W. B. and Fan, L. Q. (2009). Proteome analysis of round-headed and normal spermatozoa by 2-D fluorescence difference gel electrophoresis and mass spectrometry. Asian J. Androl. 11, 683-693.

Lin, Y. N., Roy, A., Yan, W., Burns, K. H. and Matzuk, M. M. (2007). Loss of zona pellucida binding proteins in the acrosomal matrix disrupts acrosome biogenesis and sperm morphogenesis. Mol. Cell. Biol. 27, 6794-6805.

Mandal, A., Naaby-Hansen, S., Wolkowicz, M. J., Klotz, K., Shetty, J., Retief, J. D., Coonrod, S. A., Kinter, M., Sherman, N., Cesar, F. et al. (1999). FSP95, a testis-specific 95-kilodalton fibrous sheath antigen that undergoes tyrosine phosphorylation in capacitated human spermatozoa. Biol. Reprod. 61, 11841197.

Matzuk, M. M. and Lamb, D. J. (2008). The biology of infertility: research advances and clinical challenges. Nat. Med. 14, 1197-1213.

Nakanishi, T., Ikawa, M., Yamada, S., Parvinen, M., Baba, T., Nishimune, Y. and Okabe, M. (1999). Real-time observation of acrosomal dispersal from mouse sperm using GFP as a marker protein. FEBS Lett. 449, 277-283.

Suzuki-Toyota, F., Ito, C., Toyama, Y., Maekawa, M., Yao, R., Noda, T., lida, H. and Toshimori, K. (2007). Factors maintaining normal sperm tail structure during epididymal maturation studied in $\mathrm{Gopc}^{-1-}$ mice. Biol. Reprod. 77, 71-82.
Taskiran, C., Baltaci, V. and Gunalp, S. (2006). Globozoospermia: do Ychromosome microdeletions play a role in this rare spermatogenic disorder? J. Reprod. Med. 51, 628-634.

Toshimori, K., Tanii, I., Araki, S. and Oura, C. (1992). Characterization of the antigen recognized by a monoclonal antibody MN9: unique transport pathway to the equatorial segment of sperm head during spermiogenesis. Cell Tissue Res. 270, 459-468

Xiao, N., Kam, C., Shen, C., Jin, W., Wang, J., Lee, K. M., Jiang, L. and Xia, J. (2009). PICK1 deficiency causes male infertility in mice by disrupting acrosome formation. J. Clin. Invest. 119, 802-812.

Xu, X., Toselli, P. A., Russell, L. D. and Seldin, D. C. (1999). Globozoospermia in mice lacking the casein kinase II alpha' catalytic subunit. Nat. Genet. 23, 118-121.

Yamaguchi, R., Yamagata, K., Ikawa, M., Moss, S. B. and Okabe, M. (2006). Aberrant distribution of ADAM3 in sperm from both angiotensinconverting enzyme (Ace)- and calmegin (Clgn)-deficient mice. Biol. Reprod. 75, 760-766.

Yamaguchi, R., Yamagata, K., Hasuwa, H., Inano, E., Ikawa, M. and Okabe, M. (2008). Cd52, known as a major maturation-associated sperm membrane antigen secreted from the epididymis, is not required for fertilization in the mouse. Genes Cells 13, 851-861.

Yanagimachi, R. (1994). Mammalian Fertilization. New York: Raven Press.

Yao, R., Maeda, T., Takada, S. and Noda, T. (2001). Identification of a PDZ domain containing Golgi protein, GOPC, as an interaction partner of frizzled. Biochem. Biophys. Res. Commun. 286, 771-778.

Yao, R., Ito, C., Natsume, Y., Sugitani, Y., Yamanaka, H., Kuretake, S., Yanagida, K., Sato, A., Toshimori, K. and Noda, T. (2002). Lack of acrosome formation in mice lacking a Golgi protein, GOPC. Proc. Natl. Acad. Sci. USA 99, 11211-11216.

Yildiz, Y., Matern, H., Thompson, B., Allegood, J. C., Warren, R. L., Ramirez D. M., Hammer, R. E., Hamra, F. K., Matern, S. and Russell, D. W. (2006) Mutation of beta-glucosidase 2 causes glycolipid storage disease and impaired male fertility. J. Clin. Invest. 116, 2985-2994.

Yoshida, K., Ito, C., Yamatoya, K., Maekawa, M., Toyama, Y., Suzuki-Toyota, F. and Toshimori, K. (2010). A model of the acrosome reaction progression via the acrosomal membrane-anchored protein equatorin. Reproduction 139, 533544. 


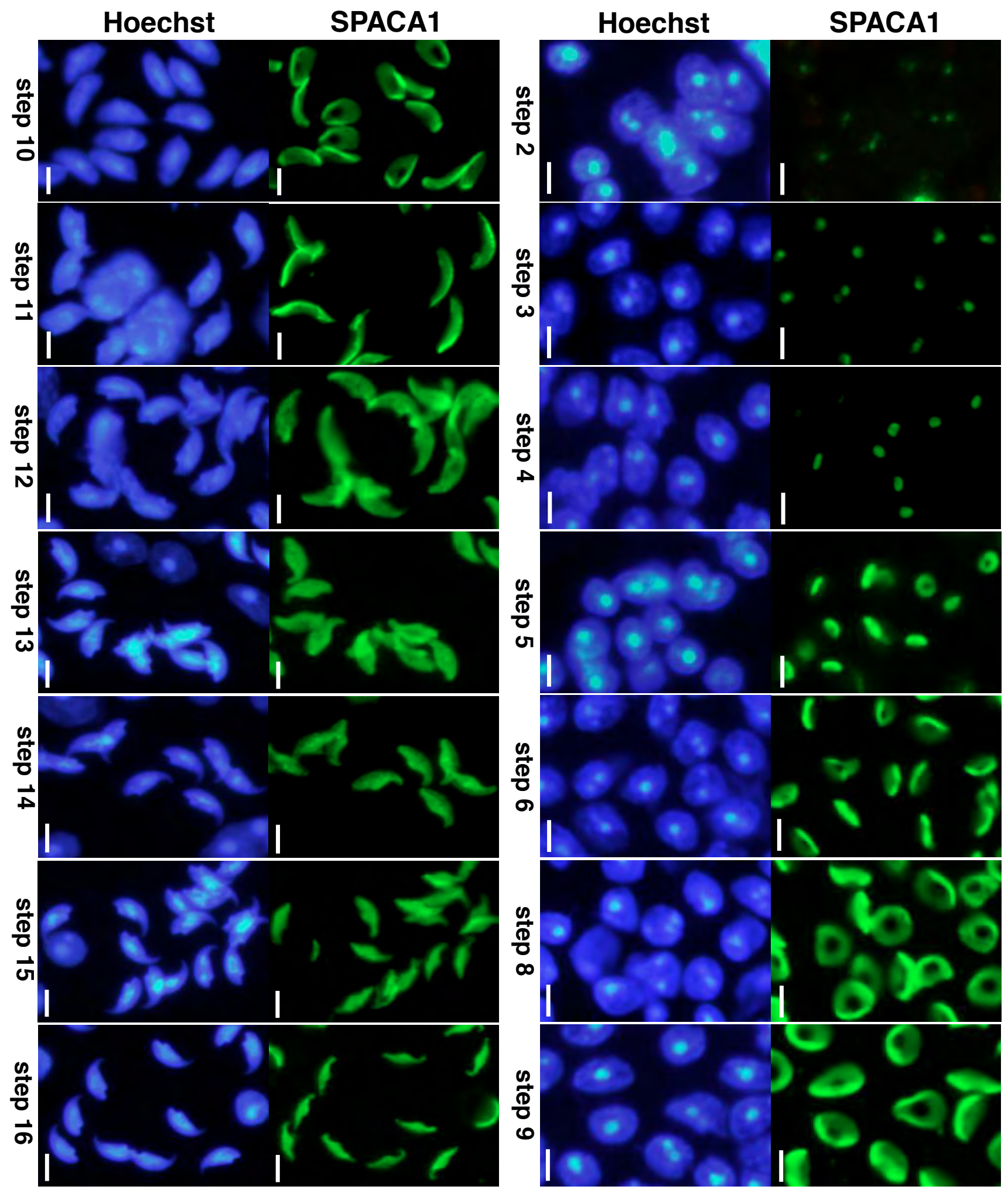

Fig. S1. Localization of mouse SPACA1 protein during spermiogenesis. Preparation of spermatogenic cells was performed as described previously (Kotaja et al., 2004). SPACA1 stained by anti-SPACA1 polyclonal antibody and immunofluorescent anti-rabbit antibody (green). Nuclei were stained with Hoechst 33342 (blue). Steps were classified by the shape of the acrosome. Scale bars: $5 \mu \mathrm{m}$. 
A

B
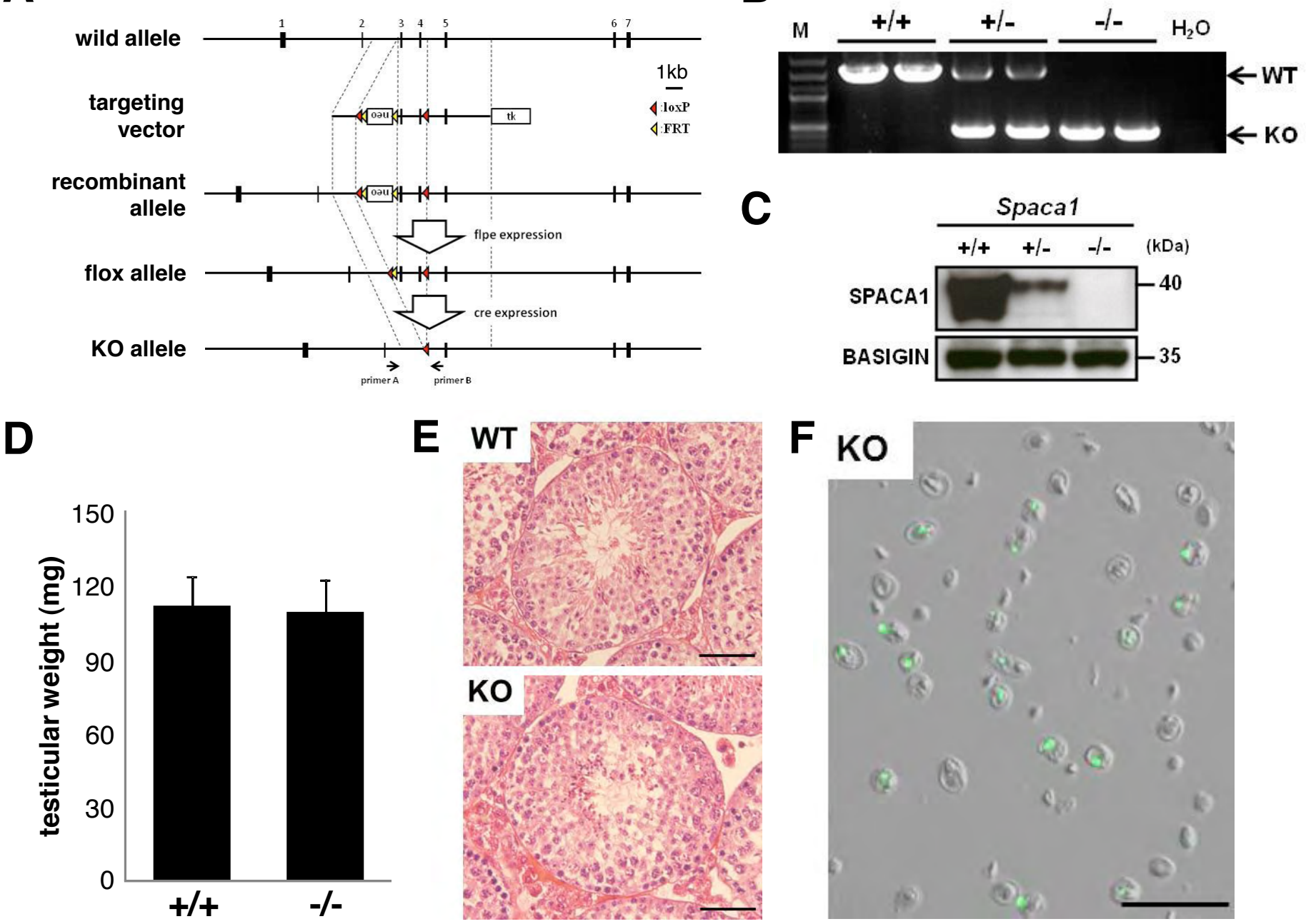

Fig. S2. Targeted disruption of the Spaca1 gene and phenotypic analysis of SPACA1-deficient mice. (A) Targeting vector to disrupt Spacal. The vector was designed to remove exons 3 and 4 of Spaca1. (B) PCR analysis detected the mutant allele in Spacal knockout mice. The upper $(1.6 \mathrm{~kb})$ and lower $(0.5 \mathrm{~kb})$ bands represent the wild-type and mutant Spacal alleles, respectively. (C) The absence of SPACA1 in testis was confirmed by western blotting. (D) Testicular weights of SPACA1-deficient males were comparable to those of wild-type males. (E) The testicular section of SPACA1deficient males did not show a severe abnormality. Scale bars: $100 \mu \mathrm{m}$. (F) All SPACA1-deficient spermatozoon were abnormal in shape in the cauda epididymis. Green fluorescence shows transgenically expressed GFP in the acrosome. Scale bar: $50 \mu \mathrm{m}$. 


\begin{tabular}{ccc}
\hline Genotype & $\begin{array}{c}\text { No. of eggs developing to } \\
\text { two-cell stage after ROSI }\end{array}$ & $\begin{array}{c}\text { No. of pups born } \\
(\%)\end{array}$ \\
\hline$+/+$ & 46 & $4(8.7)$ \\
\hline$-/-$ & 52 & $5(9.6)$ \\
\hline
\end{tabular}

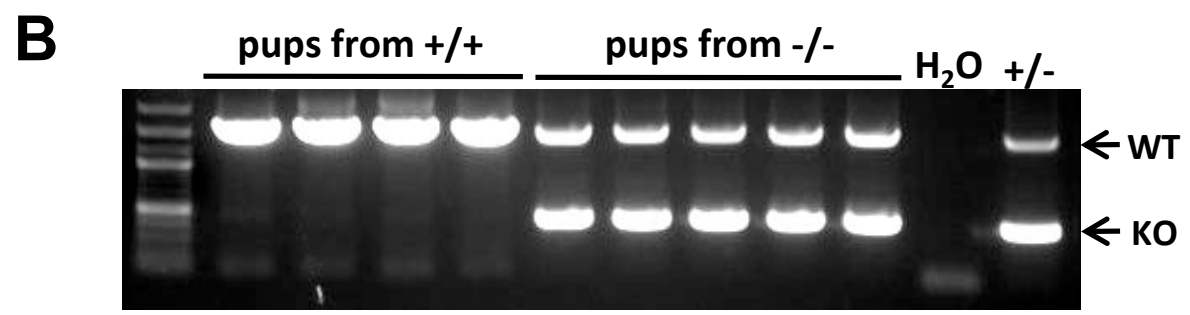

Fig. S3. Round spermatid injection (ROSI) of SPACA1-deficient spermatids. (A) Successful production of pups from SPACA1-deficient males by ROSI. The birth rate from SPACA1-deficient spermatids was comparable to that of wild-type spermatids. (B) All pups obtained by ROSI using SPACA1-deficient spermatids inherited the mutant allele as expected.
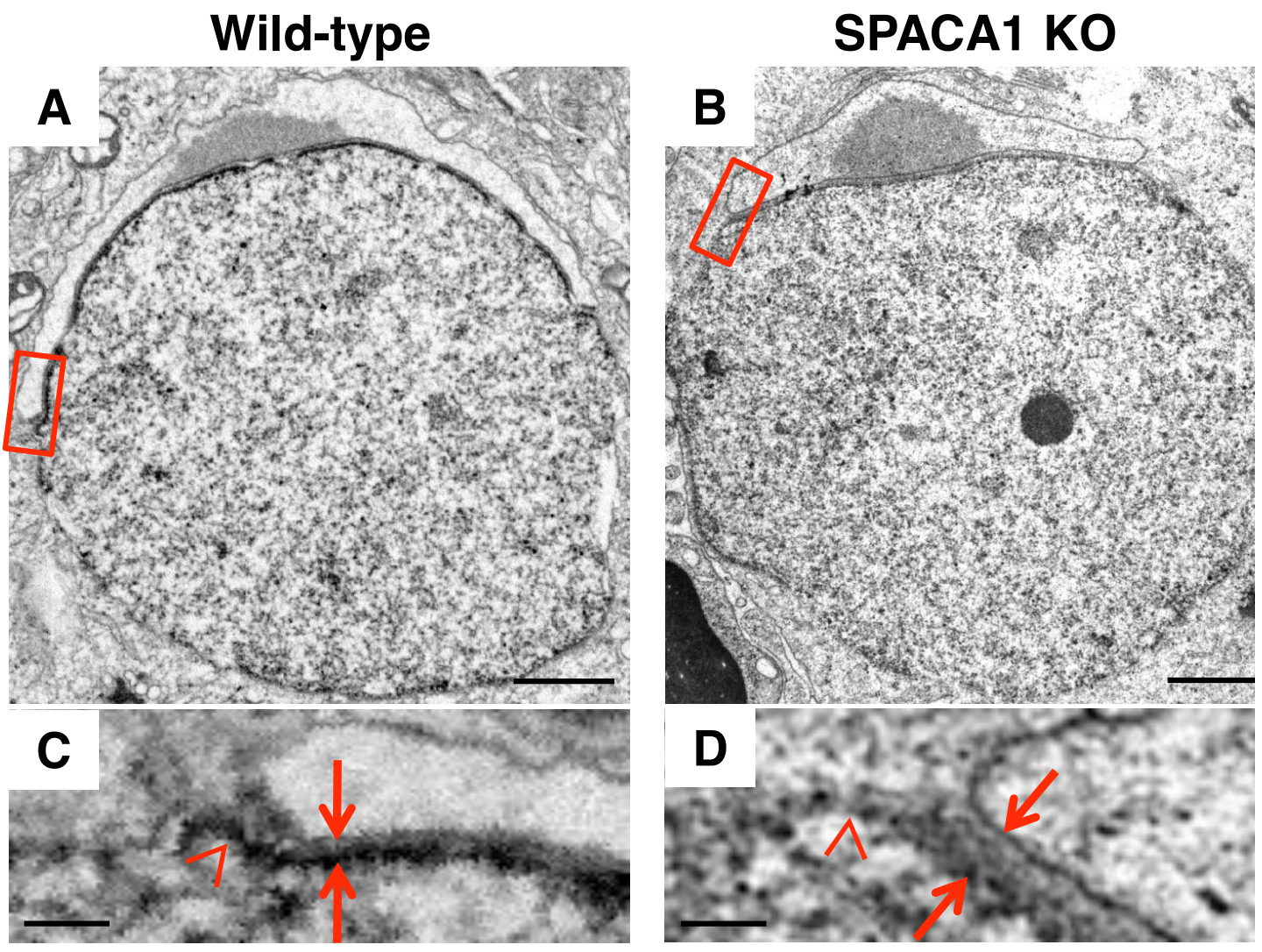

Fig. S4. TEM analysis of wild-type and SPACA1-deficient spermatids. (A,B) A comparative TEM view of wild-type (A) and SPACA1-deficient (B) spermatids. The wild-type spermatid could be judged as being at around step 7 from the extension of the acrosome, but spermatids from SPACA1-deficient mice could not be staged accurately because acrosomal formation did not proceed after step 6 . Scale bars: $1 \mu \mathrm{m}$. (C,D) The characteristic curvature (C, arrowhead) of the wildtype nuclear envelope that appears around step 7 in the spermatid. This structure might be involved in the acrosomeacroplaxome-manchette complex at the peripheral edge of the acrosome. This characteristic curvature was never formed in SPACA1-deficient spermatids (D, arrowhead). The distance between the acrosome and the nuclear membrane (between the two arrows) in SPACA1-deficient spermatid was not as tight as that of the wild-type spermatid, indicating impaired formation of the acroplaxome in SPACA1-deficient spermatids. Scale bars: $0.2 \mu \mathrm{m}$. 